\title{
Transvaginal Biopsy
}

National Cancer Institute

\section{Source}

National Cancer Institute. Transvaginal Biopsy. NCI Thesaurus. Code C159879.

Biopsy of structures in the pelvis obtained by a needle inserted through the vag inal wall. 